\title{
Experimental Identification of Friction and Its Compensation in Precise, Position Controlled Mechanisms
}

\author{
Craig T. Johnson, Member, IEEE, and Robert D. Lorenz, Senior Member, IEEE
}

\begin{abstract}
There are two primary objectives of this paper. The first is to present methodologies developed for experimentally determining accurate models for the nonlinear friction inherent in most mechanisms. The second objective is to present alternative closed-loop controller strategies for decoupling the effect of friction in order to improve positioning accuracy. The identifcation methodology is novel in the manner in which it extracts the nonlinear friction properties from the closed-loop errors via an iterative signal processing technique. The paper is based on both theoretical modeling and on a practical position control problem that was substantially resolved in developing the methodologies. The application was a robotic gripper with a highly preloaded rack-and-pinion mechanism. The paper provides both measurement and controls design methodologies to help systematically circumvent the problems of nonlinear friction in precise, position-controlled mechanisms.
\end{abstract}

\section{INTRODUCTION}

$\mathrm{F}$ RICTION EXISTS in all mechanisms to some extent. In many motion control applications, friction is a dominant factor that limits the performance of the system. However, because of its nonlinear nature, friction is often neglected or inadequately compensated by conventional controllers.

Much work has been discussed in the literature in terms of formulating a friction model, identifying its parameters, and compensating its effects [1]-[6]. Several friction models are plotted against velocity in Fig. 1. The first shows a simple "relay" characteristic to represent Coulomb or sliding friction. Breakaway friction, or stiction, is included in Fig. 1(b) as well as viscous damping. Fig. 1(c) contains an exponential model with a negative slope at low speeds [4], [5]. Furthermore, asymmetric friction properties have been documented in some applications [2]. To date, these asymmetries have normally been associated with the direction of motion.

Paper MID 92-003, approved by the Applied Automation Committee of the IEEE Industry Applications Society for presentation at the 1991 Industry Applications Society Annual Meeting, Dearborn, MI, September 28 -October 4 . This work was supported by the Wisconsin Center for Space Automation and Robotics (WCSAR) in the College of Engineering at the University of Wisconsin-Madison. Manuscript released for publication January 29, 1992.

C. T. Johnson is with Hewlett-Packard Co., San Diego, CA 92127.

R. D. Lorenz is with the University of Wisconsin-Madison, Madison, WI 53706-1691.

IEEE Log Number 9203283
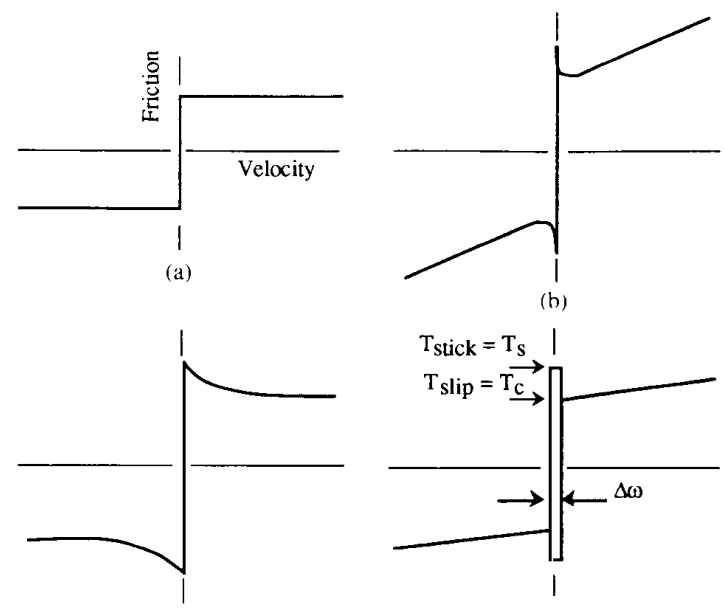

(c)

(d)

Fig. 1. Velocity-dependent friction models: (a) Columb friction model; (b) including stiction and viscous damping; (c) exponential model; (d) causal model with stick region.

A fundamental problem with these models is that they are not causal, that is, the discontinuity at zero speed allows the friction to take on an infinite number of values. This problem appears as errors or instabilities in algorithms that depend on "true" zero velocity to correctly compensate the friction. Systems that differentiate a quantized position signal to estimate velocity are especially vulnerable since a calculated zero speed may not occur during velocity reversals.

A solution to this problem is proposed in [1]. This solution circumvents the discontinuity by defining a small "stick" region at a minute speed in which the velocity is defined to be zero, as shown in Fig. 1(d). In this region $\pm \Delta \omega$, the friction force balances the net force acting on the rest of the system. Equilibrium is maintained until the breakaway force is exceeded, and the system moves into the slip region.

Although other methods exist, a crucial test of any friction model is its ability to accurately predict the friction forces near zero speed since the dynamics in this region are often dominated by the nonlinearity.

The first steps in compensating friction are formulating a feasible, physically correct model and then identifying 
its parameters. However, conventional systems identification methods have two drawbacks; most apply only to linear systems, and all require prior knowledge of the model structure. Because of the vast variations in the friction models proposed, it may be a difficult task to select the proper model structure for a particular mechanism. At the worst, an arbitrary choice is made, and the data is force fit to an inappropriate model.

The next step involves setting up experiments for identifying the model parameters. For example, it is common to measure the actuator current just before and after motion begins to estimate the breakaway and sliding friction torques, respectively. However, this technique does not take into account inertial effects, which would require knowledge of acceleration. Unfortunately, many practical motion control systems do not have the means to measure or accurately estimate acceleration without additional, expensive equipment.

Because of these limitations, a need exists for a simple method for identifying the friction nonlinearity: not only the model parameters but also their structure. This paper proposes a method that extracts the friction characteristic from the loop errors of a state feedback motion controller. The controller is initially designed by incorporating all available system knowledge into the model. The unmodeled effects, such as friction, will appear as state errors in the controller. By using signal processing analysis techniques to isolate the errors as functions of the states (e.g., velocity or position), the physical relationship between friction and the spatial states become readily apparent. This observed relationship is used to formulate an appropriate model structure. Then, these same torque errors are used to identify the model parameters. This model is then used to implement a nonlinear friction compensation in a feedforward or feedback format. By applying this method in an iterative scheme, the system's performance can be continually improved by progressively extracting and compensating the dominant, unmodeled terms until the tracking errors are reduced to an acceptable level.

Another key advantage of this strategy is that existing control hardware is usually sufficient to identify the friction model and implement its compensation. Thus, in-situ experiments can be used to improve the algorithms of existing servo systems, often without adding costly new hardware.

To demonstrate its utility, this method was applied to a robotic gripper. The results show that substantial improvements in accuracy can be obtained by minimizing the effect of friction in the mechanism.

This paper first develops the principles of the proposed method and then demonstrates its practical application.

\section{State Feedback Controller Design}

As previously stated, the proposed method uses controller errors for identifying the friction nonlinearity. Thus, a logical first step involves designing a state feedback controller or restructuring an existing controller so that the signals of interest are available for analysis. Since this method uses controller errors for identifying the friction nonlinearity, proper attention should be focused on its initial design.

There are two objectives for any motion controller; first to accurately track the commanded trajectory and second to reject all other disturbances.

The first requirement is accomplished by designing a feedforward controller to enhance tracking accuracy. A model-based feedforward controller should be used to compensate for any dynamics for which there are reasonable estimates (e.g., inertia, damping, back-emf, friction, etc.). The state equation describing a linear system with input $U$ is

$$
\dot{X}=A X+B U
$$

For a tracking controller, $X=X^{*}$ is desired, where $X^{*}$ is a vector of the commanded states. Substituting this into the state equation, the feedforward control inputs $U_{f f}$ must be

$$
U_{f f}=\hat{B}^{-1}\left[\dot{X}^{*}-\hat{A} X^{*}\right]
$$

where $\hat{B}$ and $\hat{A}$ are estimates. A block diagram for a torque-controlled servomotor-driven mechanism with total reflected inertia $J$ and viscous damping $b$ is shown in Fig. 2. A high bandwidth cascaded current loop and field-oriented control are assumed; therefore, the electrical dynamics can be neglected. For this model, the feedforward torque command for ideal tracking would be [7], [8]

$$
T_{f f}=\hat{J} \dot{\omega}^{*}+\hat{b} \omega^{*}
$$

where $\hat{J}$ and $\hat{b}$ are estimates of $J$ and $b$, respectively. It will be shown later that errors in the estimates of the feedforward parameters can be tolerated, but even partial cancellation of known system dynamics will improve the initial results.

The second controls objective is disturbance rejection. This criteria is accomplished by state feedback as shown in Fig. 3, where state feedforward is also included. The ability of a controller to attenuate extraneous loads can be described by the controller's "dynamic stiffness" [9], [10]. This measure of disturbance rejection can be derived as

$$
\frac{T_{d}(s)}{\theta(s)}=J s^{2}+\left(b_{a}+b\right) s+K_{a} .
$$

Note that the state feedback gains of this topology take on physical significance. For example, the position gain behaves like an "active" spring with stiffness $K_{a}$ and units $(\mathrm{N}-\mathrm{m} / \mathrm{rad})$. Similarly, the velocity gain has properties and units of damping, but it is "active" because it does not dissipate power in heat. When multiplied by their respective state errors, a feedback torque error that attempts to correct the physical states is produced:

$$
T_{f b}=K_{a}\left(\theta^{*}-\theta\right)+b_{a}\left(\omega^{*}+\omega\right) .
$$




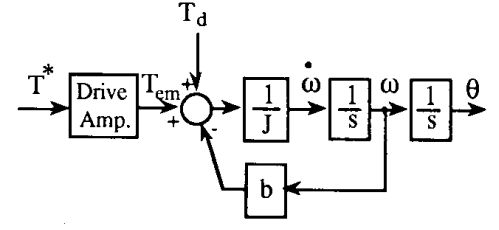

Fig. 2. Block diagram of a torque-controlled motor driven load.

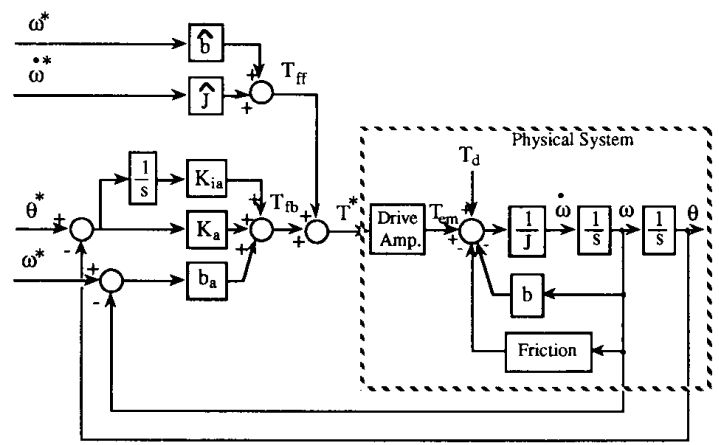

Fig. 3. Block diagram of a motion-controlled system with feedforward and linear state feedback control.

The topology of Fig. 3 provides an important functional distinction between feedforward and feedback. The feedforward controller provides accurate command tracking, whereas the feedback controller attempts to reject disturbances in the form of external loads or unmodeled dynamics. In doing so, it acts as a disturbance estimator. Thus, the poles of the controller should be placed to obtain disturbance rejection properties, whereas the model-based feedforward controller should be independently designed for command following.

The use of a state integrator should also be noted in Fig. 3. This integrator is highly desirable in most position control systems. However, it is well documented that integral controller states can cause limit cycles and/or instability in the presence of friction. The stick-slip nature of friction works against the tendency of the integrator to drive steady-state errors to zero. This antagonizing relationship renders integral control unfit for controlling systems with substantial friction. Of course, without integral control, relatively large, steady-state errors can remain uncompensated. Thus, a major motivation for decoupling the friction nonlinearity is to produce a system with predictable, stable, space-invariant dynamics.

\section{An Identification Method Based on State Feedforward, State FeEdBack Properties}

The state feedforward, state feedback controller developed in the last sections serves as the foundation of the proposed friction identification method. Its use and an introduction to the methodology will be based on the following analysis (based on a simulation of the system in Fig. 3).

\section{A. An Iterative Systems Identification Methodology}

Suppose the feedforward is ideal $(\hat{J}=J$ and $\hat{b}=b)$. Then, the system will track the commands perfectly with zero state errors. However, if the feedforward model does not accurately represent the physical system, tracking errors will result. As previously stated, unmodeled dynamics of the system will appear as feedback torque commands $T_{f b}$, as described in (5), that is, the unknown friction torque in the mechanism must be contained in $T_{f b}$.

As a systems test procedure, assume that the system can be driven over trajectories that includes a velocity reversal. A possible trajectory state command set is shown in Fig. 4. If the feedback torque error $T_{f b}$ is measured during the trajectory and plotted verses velocity $\omega$, the result may look similar to one of the cases shown in Fig. 5. Here, feedforward has cancelled all dynamics except for the system friction, and torque error is entirely caused by the physical friction. However, it is more likely that the feedforward compensation is not perfectly accurate, and errors due to inertia and damping will also be present. Fig. 5(a) represents a worse case since no feedforward compensation is used (which implies $\hat{J}=0, \hat{b}=0$ ). The basic friction characteristic is present, but it is contaminated by other errors. For example, the hysteresis loop of Fig. 5(a) is caused by inertial errors and will be present whenever the system is accelerating. The size of the hystersis loop can be used to estimate the inertia of the system

$$
\hat{J}=\frac{\Delta T_{f b}}{2} \frac{1}{\dot{\omega}^{*}}
$$

where $\Delta T_{f b}$ is the width of the loop. Note that the inertia estimate could be obtained from a plot of $T_{f b}$ versus $\dot{\omega}$ if a good measure of acceleration were available.

After a good estimate of inertia is made, it will be added to the feedforward path to reduce or eliminate state errors while accelerating. With the improved tracking control, the system is again exercised over the same trajectory. The new $T_{f b}$ versus $\omega$ relationship has no hysteresis loop if the inertial compensation was successful, as observed in Fig. 5(b).

The sloped line in the $T_{f b}$ that is characteristic of Fig. 5(b) suggests that a viscous damping estimate is also present. The slope of this line allows the damping coefficient $\hat{b}$ to be estimated directly by standard least squares curve fitting.

It is appropriate to note that the sloped $T_{f b}$ versus $\omega$ characteristic can also be caused by back emf in systems that use voltage regulation rather than current regulation in the power converter. In either case, it can be identified and compensated using the same method.

At this point, the feedforward terms include accurate inertial and viscous damping terms. Repeating the command trajectory, the friction characteristic of Fig. 5(c) is observed. The two parameters that define the friction model $T_{\text {stick }}=T_{s}$ and $T_{\text {slip }}=T_{c}$ are obtained by inspec- 


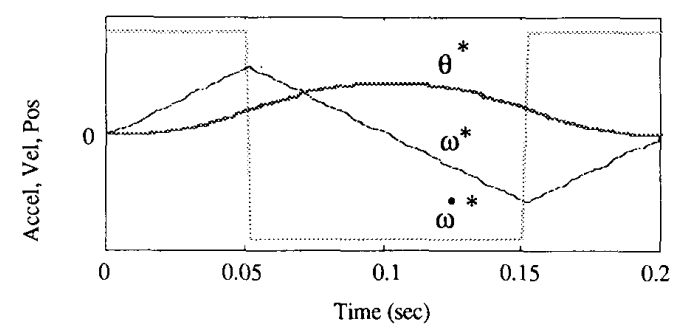

Fig. 4. Complete command set for experimental trajectory.

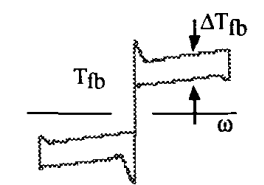

(a)

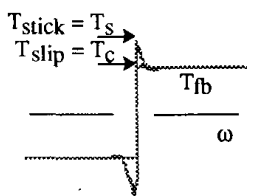

(c)
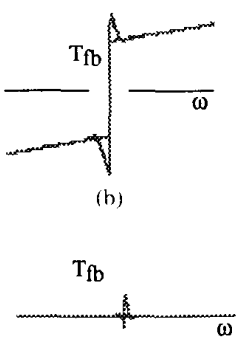

(d)
Fig. 5. Feedback torques versus velocity with progressively improved feedforward model (robot gripper model): (a) No feedforward; (b) inertia compensated; (c) viscous damping compensated; (d) stiction and sliding friction compensated.

tion. Incorporating these parameters in the feedforward model yields complete friction compensation, with only residual torque feedback noise remaining, as shown in Fig. 5(d).

Summarizing this method, a feedforward model is built by successively extracting the uncompensated system dynamics from the feedback errors. Proper construction of the controller assigns units of torque to these feedback errors. Viewing the controller errors as torques allows direct correlation to physical phenomena, such as inertial, damping, and friction torques. Plots of $T_{f b}$ versus velocity show the structure of the friction characteristic and provide response data for estimating its parameters. As the characteristics are identified, their effects are included in the compensation algorithm. This process is pursued iteratively until tracking errors are reduced to an acceptable level. This methodology can be extended to expose spatial dependencies such as robotic link gravitational torques, which would be identified on a $T_{f b}$ versus position plot.

\section{B. The Use of Spatial Synchronous Averaging}

A potential problem with any identification method is the contamination of data by uncorrelated signals inherent in the data. Most methods attempt to reduce the effects of such unwanted signals by the selection of robust signal processing techniques. One signal processing technique (synchronous averaging) has proven particularly ef- fective in attenuating such noise during the experimental stage.

Most averaging methods are based on attenuating signals that are not correlated with the common reference variable (usually time). Random noise is an example of such a signal that is asynchronous and uncorrelated in time. However, other types of noise can still taint the results due to their correlation to the common reference. Examples include other physical characteristics that vary with position, acceleration, or current (e.g., gravitational loads, torque ripple, spatially varying loads, Coriolis effects, etc.). These unknown physical effects are unmodeled so they will also be contained in the $T_{f b}$ signal, along with the frictional properties of interest as shown by (7).

$$
\begin{aligned}
T_{f b}(\dot{\omega}, \omega, \theta, i, t) & \\
= & T_{\text {friction }}(\omega)+T_{\text {other }}(\dot{\omega}, \omega, \theta, i, t) .
\end{aligned}
$$

Thus, to improve the friction model, it is desirable to attenuate the components of $T_{f b}$ that are asynchronous (uncorrelated) with the spatial variable, velocity (i.e., $T_{\text {other }}$ ). This will leave only the desired signal that is synchronous with velocity, i.e., $T_{\text {friction }}$. By proper design of the experiment, a deterministic effort is made to average out the signals that are asynchronous with the spatial variables, most especially with velocity.

This concept suggests that data be averaged in the velocity (not time) domain. A common velocity distribution should be used in all experiments, but all other motion variables are varied in order to average out effects not included in the velocity-dependent friction model. A number of velocity trajectories can be generated, where each has the same shape and size, but the corresponding position and acceleration trajectories can have different magnitude, direction, and initial positions. Fig. 6 shows a set of possible test trajectories. The fact that total motion time is being varied from one trajectory to the next (i.e., slow trajectories take longer to complete than fast ones) is implicit in the horizontal axis of Fig. 6. Thus, undesirable spatial and temporal dependencies can both be averaged out at the same time.

\section{Experimental Results: Spatial Synchronous Averaging}

The actual averaging algorithm is unique because the measured torque errors need to be converted to a velocity reference frame before point-by-point averaging, that is, discrete velocity points must be chosen and the torque errors averaged only at those points. One problem is that the data obtained from the digital controller consists of discrete data points at uniformly spaced times. In this work, the fastest trajectory was used as the velocity reference. It uses the closest velocity points in all slower trajectories. Intermediate points were discarded. Linear interpolation was used to interpolate the torque errors at the corresponding velocity reference points. Decimating the torque errors in this way converts the data to a form suitable for averaging in the velocity domain. Experimental results in Fig. 7 show the final friction characteristic 


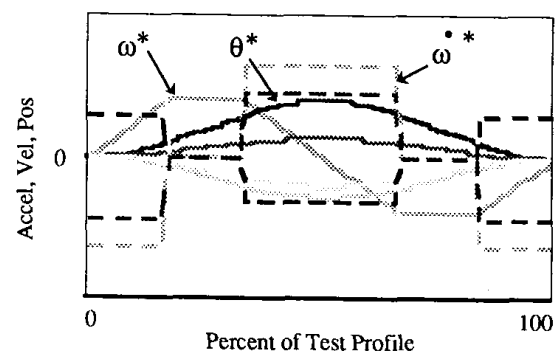

Fig. 6. Set of trajectories used for synchronous averaging. Note that velocity distribution is the same in all trials.

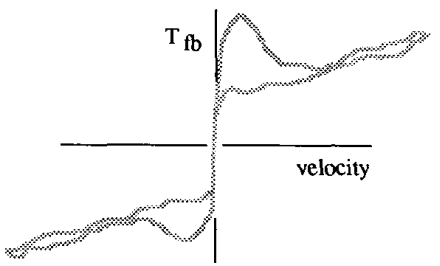

Fig. 7. Experimental controller torque errors after synchronous averag ing.

for the robot gripper smoothed by such synchronous averaging.

Some observations can be made from the acquired data. Close inspection of Fig. 7 shows a slight asymmetry between the right and left sides of the characteristic. This property was found to be temporally related and not spatially related, that is, the static breakaway friction was observed to increase with time at rest, regardless of the direction of motion. Thus, the starting direction after stoppages will affect the polarity of this effect.

This time-dependent friction force appears to be related to lubrication voiding and creep. On a macro scale, lubricants will tend to be squeezed out between two surfaces under load. On a micro scale, the area of contact will increase by creep under load. As more time is available, more asperities become interlocked at the interface, and the force required to begin motion increases. According to [11], a few seconds at rest are required to reach the maximum static friction force. This helps explain why the static torque is lower during short velocity reversals within a trajectory.

A second issue observed in the experimental work was the overshoot and dynamic response at low speeds just after breakaway. This "looping" characteristic did not prove to be a component of the friction model being estimated. In fact, it was discovered that the system was underdamped, and the overshoot could be controlled by increasing the active damping $b_{a}$. Even though the closed-loop poles were placed for critical damping, the physical system dynamics were affected by friction, resulting in a nonlinear, space-variant response. This observation was verified by simulation. Fig. 8 shows the simulated gripper response to the friction characteristic in Fig. 1(c)

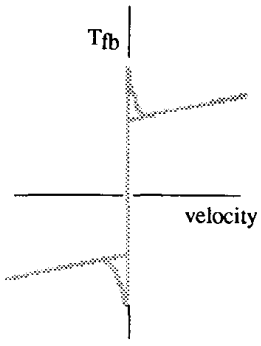

(a)

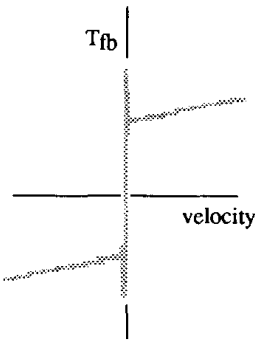

(b)
Fig. 8. Using active damping to attenuate controller dynamic response: (a) $b_{a}=b_{c r i t}$; (b) $b_{a}=4 * b_{\text {crit }}$.

as the active damping gain is increased by four times critical damping. It should be recognized that the gain (active damping) achievable in the actual system was limited by the sampling rate and feedback signal quantization of the digital controller. Regardless of the damping level achieved, it is important to not mistake the controller dynamics for physical, friction phenomena. This is a potential hazard when using a linear controller to control a nonlinear system.

One last comment is appropriate concerning identification. It may seem reasonable to evaluate only the controller torque signal produced by velocity errors (i.e., $\left.b_{a}\left(\omega^{*}-\omega\right)\right)$ since we are trying to average out position dependencies. Fig. 9 is a breakdown of the subcomponents comprising the $T_{f b}$ signal. Clearly, neither signal is useful for identification purposes by itself. The composite signal contains the information needed for parameter estimation.

\section{FRICTION COMPENSATION}

With the frictional characteristic of the mechanism accurately identified, two nonlinear control compensation methods will be compared. Nonlinear decoupling state feedback (FB) compensation decouples the friction nonlinearity in the closed loop by using measured states to calculate the friction model. In contrast, the nonlinear decoupling state feedforward (FF) compensation method utilizes commanded states. Figs. 10 and 11 show the two controller topologies.

\section{A. Nonlinear State Feedback}

Feedback compensation implements the causal friction model described in Fig. 1(d)) with stick region width $\pm \Delta \omega$ :

$|\omega|>\Delta \omega \quad$ (system is in the "slip" region)

$$
\hat{T}_{\text {friction }}=\hat{T}_{c} \cdot \operatorname{sign}(\omega)+b \omega
$$

$|\omega|<\Delta \omega \quad$ (system is in the "stick" region)

$$
\hat{T}_{\text {friction }}=\operatorname{MIN}\left\{\hat{T}_{e m}, \hat{T}_{s} \cdot \operatorname{sign}\left(\hat{T}_{e m}\right)\right\}
$$

where the MIN function here selects the number closest to zero and limits the friction torque to the breakaway 


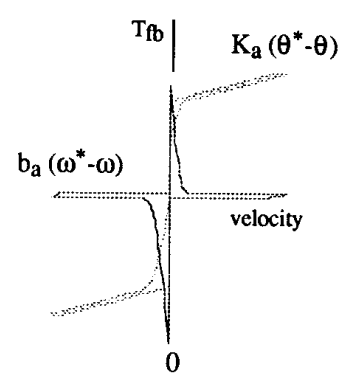

Fig. 9. Breakdown of feedback torque error components.

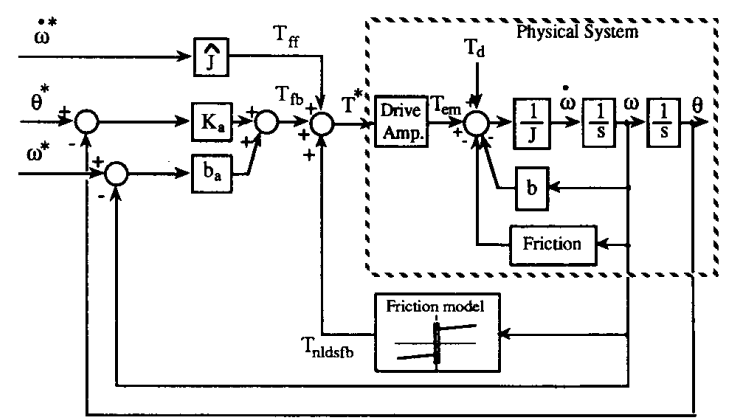

Fig. 10. Nonlinear decoupling state feedback friction compensation technique.

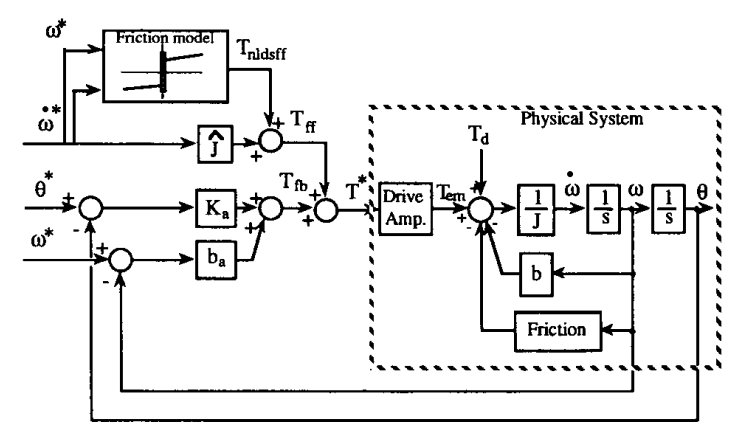

Fig. 11. Nonlinear decoupling state feedforward friction compensation technique.

value $T_{s}$ in the stick region. $\hat{T}_{\text {friction }}$ is the calculated friction torque used for the compensation, and $\hat{T}_{e m}$ is the estimated electromagnetic torque produced by the motor. From the block diagram in Fig. 10, the controller output is a good estimate of $T_{e m}$, that is, the sum of feedback and feedforward torque corrections

$$
T_{e m}=T_{f f}+T_{f b} .
$$

The quality of the $T_{e m}$ estimate is good if the assumption of a high bandwidth torque control loop is satisfied and if the motor torque is accurately known. In the case of the robot gripper, the amplifier dynamics are an order of magnitude faster than the closed-loop mechanical poles; therefore, the assumption of a unity-gain, cascade torque regulator seems reasonable. A velocity estimate is also required for the digital controller and is obtained by a direct difference approximation of the encoder signal

$$
\hat{\omega}(k)=\frac{\theta(k)-\theta(k-1)}{T}
$$

where $T$ is the sampling period. The differential estimate proved to be fairly smooth in this application because the encoder's resolution (4096 ppr) was high relative to the sampling frequency.

\section{B. Nonlinear State Command Feedforward}

The feedforward controller uses a friction model similar to (8) but with some modifications. By definition, commanded states are used in place of the actual states. In addition, since no estimate of the feedback $T_{f b}$ is available, the $T_{e m}$ estimate must be derived completely from feedforward (i.e., $\hat{T}_{e m}=T_{f f}$ ).

\section{EXPERIMENTAL RESULTS}

Fig. 12 compares the results of feedback, feedforward friction compensation relative to the uncompensated gripper. Both the feedforward and feedback implementations produced a substantial reduction in position and velocity errors over the initial system without friction compensation. For the robot gripper, position tracking errors were reduced by a factor of four or more when the friction compensation was added. The feedforward and feedback implementations produced similar results over most of the trajectory. However, the feedback friction compensation performed best in the regions near zero velocity, which resulted in smaller steady-state (endpoint) errors. In fact, the endpoint position errors were reduced twofold over the feedforward compensation and tenfold over the controller without friction compensation.

The two methods can be compared meaningfully at this point. The feedforward implementation precomputes the friction estimate based on the command vector. This makes the feedforward strategy almost "free," since no additional closed-loop computation is required to compensate friction. In most cases, even more complex frictional models, such as asymmetrical frictional effects, can be incorporated at no additional real-time cost.

However, the feedforward method is fundamentally limited by its use of the command vector. Herein lies the key advantage of feedback compensation; actual states are used to calculate the friction correction. If the model is accurate (i.e., $\hat{T}_{c}=T_{c}, \hat{T}_{s}=T_{s}$ ), it is then theoretically possible to linearize the system, that is, by decoupling the friction nonlinearity with state feedback, we can cancel the space-variant dynamics. This attribute makes the feedback compensated system more robust to other disturbances than its feedforward counterpart. However, Figs. 10 and 11 imply that this advantage has a cost associated with the additional real-time computational burden of the feedback compensation terms.

Clearly, there is a tradeoff between the quality of the friction compensation and the computational cost of im- 

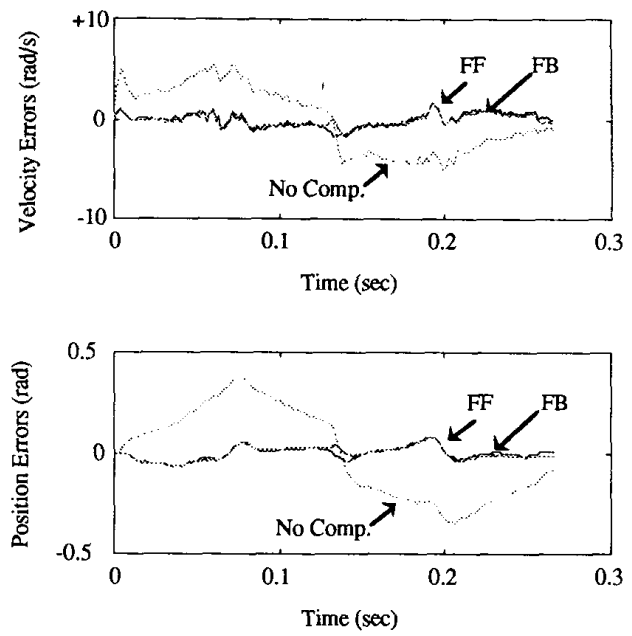

Fig. 12. State errors for three controllers; friction compensation in feedback, feedforward, and uncompensated: (top) velocity errors; (bottom) position errors.

plementing it. Both should be evaluated in light of the economics of a particular application.

\section{CONCLUSIONS}

An experimental methodology for identifying nonlinear friction models in mechanisms has been developed and presented. Its key attributes are as follows:

- It is an integrated signal processing and experimental methodology.

- It extracts the frictional properties from the existing controller torque errors if the controller is properly formed to include both state feedforward and state feedback.

- It does not generally require the use of extra sensors other than those already used for the motion controller.

- It extracts robust estimates by using spatial synchronous averaging on the modeled variable. Two alternative friction compensation controller alternatives have been developed and evaluated.

- Both nonlinear state command feedforward and nonlinear state feedback techniques have demonstrated substantial improvement in positioning accuracy for a robotic gripper application.

- Nonlinear decoupling state feedback is the most robust, but it is generally more costly to implement due to the higher real-time computation needs.

- Nonlinear decoupling state feedforward is less robust, but it does perform well for the friction compensation needed in this robotic gripper application.

\section{REFERENCES}

[1] D. Karnopp, "Computer simulation of stick-slip friction in mechanical dynamic systems," J. Dyn. Syst. Meas. Contr., vol. 107, Mar. 1985.
[2] V. Held and C. Maron, "Estimation of friction characteristics, inertial and coupling coefficients in robotic joints based on current and speed measurements," in Proc. IFAC Robot Contr. (Karlsruhe, FRG), 1988.

[3] T. Kubo, G. Anwar, and M. Tomizuka, "Application of nonlinear friction compensation to robot arm control," in Proc. Int. Conf. Robotics Automat. (San Francisco), 1986.

[4] P. R. Dahl, "Measurement of solid friction parameters of ball bearings," in Proc. 6th Ann. Symp. Incremental Motion Contr. Syst. Devices (Univ. Illinois, Chicago), 1977.

[5] C. D. Walrath, "Adaptive gearing friction compensation based on recent knowledge of dynamic friction," Automatica, vol. 20, pp. $717-727,1984$.

[6] C. Canudas, K. J. Astrom, and K. Braun, "Adaptive friction compensation in dc-motor drives," J. Robotics Automat., vol. RA-3, no. 6, Dec. 1987.

[7] R. D. Lorenz, J. J. Zik, and D. J. Sykora, "A direct-drive, robot parts and tooling gripper with high performance force feedback control," in IEEE Trans. Industry Applications, vol. 27, no. 2, pp. 275-282, Mar./Apr. 1991.

[8] R. D. Lorenz and P. B. Schmidt, "Synchronized motion control for process automation," in IEEE-LAS Conf. Rec. 1989, pp. 1693-1698.

[9] R. D. Lorenz, "Synthesis of state variable controllers for industrial servo drives," in Proc. Conf. Appl. Motion Contr., June 1986, pp. 247-251.

[10] G. W. Younkin, W. D. McGlasson, and R. D. Lorenz, "Considerations for low inertia ac drives in machine tool axis servo applications," in IEEE Trans. Industry Applications, vol 27, no. 2, pp. 262-268, Mar./Apr. 1991.

[11] A. D. Sarkar, Friction and Wear. New York: Academic, 1980.

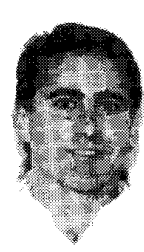

Craig T. Johnson (M'92) received the B.S. degree in mechanical engineering from the University of Minnesota in 1985 and the M.S. degree in electrical and computer engineering from the University of Wisconsin-Madison.

He has worked in new product development at Hewlett-Packard in San Diego, CA, for six years. His current interests include electromagnetic machines, control systems, and power electronics.

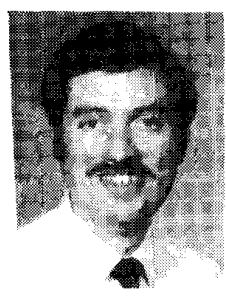

Robert D. Lorenz (M'84-SM'91) received the B.S., M.S., and Ph.D. degrees, all in mechanical engineering (electromechanics and control specialty) from the University of Wisconsin-Madison in 1969,1970 , and 1984 , respectively.

Since 1984 , he has been a member of the faculty of the University of Wisconsin-Madison, where he is an associate professor of mechanical engineering and of electrical and computer engineering. In this position, he acts as Associate Director of the Wisconsin Electric Machines and Power Electronics Consortium and as Co-Director of the Advanced Automation and Robotics Consortium. He was a Visiting Research Professor in the Electrical Drives Group of the Catholic University of Leuven, Leuven, Belgium, and in the Electrical Drives Institute of the Technical University of Aachen, West Germany, in the summers of 1991 , 1989, and 1987, respectively. During 1969-1970, he did his Master thesis research at the Technical University of Aachen, West Germany. From 1972 to 1982 , he was a member of the research staff at the Gleason Works, Rochester, NY. His current research interests include optical and electromagnetic sensor technologies, real-time digital signal processing techniques, electromagnetic actuator design and control, and ac drive and high-precision machine control technologies.

Dr. Lorenz is Chairman of the IAS Industrial Drives Committee and is a member of the Applied Automation Committee and the Industrial Control Committee. He is an active consultant to many organizations and is a Registered Professional Engineer in the States of New York and Wisconsin. He is a member of ASME, ISA, and SPIE. 